\title{
Distribution of Molecular Gas in Barred Spiral Galaxies
}

\author{
N. Kuno ${ }^{1,2}$, N. Sato ${ }^{3}$, H. Nakanishi ${ }^{1}$, A. Hirota ${ }^{4}$, T. Tosaki ${ }^{1}$, \\ Y. Shioya ${ }^{5}$, K. Sorai ${ }^{6}$, N. Nakai ${ }^{7}$, K. Nishiyama ${ }^{8}$, and B. Vila-Vilaró ${ }^{9}$ \\ ${ }^{1}$ Nobeyama Radio Observatory, Minamimaki-mura, Minamisaku-gun, Nagano 384-1305, Japan \\ ${ }^{2}$ The Graduate University for Advanced Studies (SOKENDI), 2-21-1 Osawa, Mitaka, Tokyo \\ 181-0015, Japan \\ ${ }^{3}$ Wakayama University, Wakayama 640-8510, Japan \\ ${ }^{4}$ The University of Tokyo, Bunkyo-ku, Tokyo 113-0033, Japan \\ ${ }^{5}$ Ehime University, Matsuyama 790-8577, Japan \\ ${ }^{6}$ Hokkaido University, Sapporo 060-0810, Japan \\ ${ }^{7}$ University of Tsukuba, Ten-nodai, 1-1-1 Tsukuba, Ibaraki 305-8577, Japan \\ ${ }^{8}$ Bisei Spaceguard Center, Bisei-cho, Oda-gun, Okayama 714-1415, Japan \\ ${ }^{9}$ National Astronomical Observatory of Japan, 2-21-1 Osawa, Mitaka, Tokyo 181-0015, Japan
}

We made a $\mathrm{CO}(1-0)$ mapping survey of 40 nearby spiral galaxies with the Nobeyama 45-m telescope to provide useful data for detailed and systematic studies of molecular gas in the galaxies (Kuno et al. 2006). Using these data we have compared the distribution of molecular gas in barred and non-barred spirals and investigated the influence of the bar. We confirmed that the degree of the central concentration of molecular gas within the radial distances of the order of a bar length in barred spirals is significantly higher than that in non-barred spirals as shown by Sakamoto et al. (1999) and Sheth et al. (2005). This is contrast with the degree of the concentration of the total molecular gas mass within the radial distances of the order of the bar, which is similar for both barred and non-barred spirals. This implies that the bars appear to be efficient in driving gas that lies within their radial scales toward the center of the host galaxies, but that they play quite a smaller role at larger spatial scales on the disks. Thus the characteristic feature of the radial distribution of molecular gas seen in barred spirals, i.e. the strong intensity peaks at their centers, the shallow gradients within the bar regions or/and the secondary peaks at the radius of the bar-ends, can be explained by the accumulation of molecular gas within the bar regions. The accumulated gas by bars accounts for about half of molecular gas mass within the central region. We also found a correlation between the degree of central concentration of molecular gas and the bar strength. Galaxies with stronger bars tend to have higher central concentrations. The result indicates that stronger bar accumulate molecular gas toward the center more efficiently. The correlation between the degree of central concentration of molecular gas and the strength seems to be consistent with long-lived bars rather than short-lived ones which are destroyed by the gas accumulation toward the center many times in the Hubble time.

\section{References}

Kuno, N., Sato, N., Nakanishi, H., Hirota, A., Tosaki, T., Shioya, Y., Sorai, K., Nakai, N., Nishiyama, K., \& Vila-Viraó. 2006, submitted to PASJ.

Sakamoto, K., Okumura, S.K., \& Ishizuki, S. 1999, ApJ 525, 691.

Sheth, K., Vogel, S. N., Regan, M. W., Thornley, M. D., \& Teuben, P. J. 2005, ApJ 632, 217. 